\title{
Omega-3 and Omega-6 Determination in Nile Tilapia's Fillet Based on MicroNIR Spectroscopy and Multivariate Calibration
}

\author{
Dayane A. dos Santos, ${ }^{a}$ Aline Coqueiro, ${ }^{\oplus b}$ Thays $R$. Gonçalves, ${ }^{a}$ Jonathan C. Carvalho, ${ }^{a}$ \\ Jailton S. Bezerra Jr., ${ }^{a}$ Makoto Matsushita, ${ }^{a}$ Carlos A. L. de Oliveira, ${ }^{a}$ Paulo H. Março, ${ }^{b}$ \\ Patrícia Valderrama ${ }^{\circledR *, b}$ and Ricardo P. Ribeiro ${ }^{*, a}$ \\ ${ }^{a}$ Universidade Estadual de Maringá (UEM), Av. Colombo, 5790, 87020-900 Maringá-PR, Brazil \\ ${ }^{b}$ Universidade Tecnológica Federal do Paraná (UTFPR), Via Rosalina Maria dos Santos 1233, \\ 87301-899 Campo Mourão-PR, Brazil
}

\begin{abstract}
Tilapia (Oreochromis niloticus) is the most cultivated fish species in Brazil. Studies have shown that fish consumption is related to the prevention of cardiovascular diseases due to its high contents of polyunsaturated fatty acids, as omega-3 (n-3) and omega-6 (n-6). In this sense, a new method to determine omega- 3 and omega- 6 by near-infrared (NIR) spectroscopy and multivariate calibration based on partial least squares (PLS) was proposed. Spectra of fillets of Nile tilapias packed under vacuum were obtained and compared to the reference method (gas chromatography) used for quantification of fatty acids. The outliers were evaluated in the models that were validated by calculation of the parameters for model performance estimation, showing promising results. The appropriate values achieved for accuracy, limits of detection and quantification, indicate that the NIR/PLS models can be an alternative to chromatography in the determination of omega-3 and omega-6 in fillets of Nile tilapia. Furthermore, the spectra acquisition on the vacuum-packed fillet contributes to the NIR/PLS method in terms of cost and quickness.
\end{abstract}

Keywords: Nile tilapia, lipids, NIR, PLS, validation

\section{Introduction}

The aquaculture provides more than half of the total fish for human consumption worldwide. It is the main responsible for the significant growth of fish production in recent years. ${ }^{1}$ In Brazil, currently, the most cultivated species is tilapia (Oreochromis niloticus). ${ }^{2}$ According to Food and Agriculture Organization (FAO), ${ }^{3}$ the tilapia production will grow even more and by 2030 , the tilapia, carp, and catfish will represent around $62 \%$ of total aquaculture production in the world.

Studies have shown that fish consumption is related to the prevention of cardiovascular diseases, ${ }^{4}$ due to its high contents of polyunsaturated fatty acids (PUFA), as omega-3 (n-3), ${ }^{4-6}$ and omega-6 (n-6), while there are recommendations of omega-6/omega-3 ratio in the diet, proposed by several countries, showing a coverage range from 4 to $5: 1 .^{7}$

*e-mail: pativalderrama@gmail.com, patriciav@utfpr.edu.br; rpribeiro@uem.br
Belonging the PUFAs family, the $n-3$ fatty acids (FA) may be used in the treatment of inflammatory diseases because they promote alteration of the cytokine biosynthesis. They also show anti-carcinogenic effects, and the n-3 FA from fish can provide the best example of functional food ingredients and nutraceuticals. ${ }^{8,9}$

The main analytical method for omegas determination is gas chromatography with flame ionization detection (GC-FID). However, this analytical technique requires several steps of sample preparation by using solvents and generates toxic waste. This justifies the researches for alternatives methodologies, as by using near-infrared (NIR) spectroscopy, for example.

NIR instrument has undergone several improvements since the beginning of its employment in the analytical field. Undoubtedly, one of the biggest was miniaturization. Some applications of portable NIR instrument in the food analysis include: determination of the sensory attributes in Brazilian coffee blends, ${ }^{10}$ quality control of parameters in the roasted coffee industry, ${ }^{11}$ fast discrimination of milk contaminated with Salmonella sp., ${ }^{12}$ discrimination of honey samples, ${ }^{13}$ 
evaluation of the thermal rice oil degradation, ${ }^{14}$ and assessment of quality parameters in ground soybean. ${ }^{15} \mathrm{In}$ this sense, the main objective of this research is to propose an alternative analytical methodology to quantify omega-3 and omega-6 in Nile tilapia by a portable NIR instrument coupled with multivariate calibration by partial least squares (PLS). Furthermore, one of the crucial aspects of novelty in this work is the employment of the portable instrument in order to quantify fatty acids on packaged fillet in a non-destructive way.

\section{Experimental}

\section{Samples}

The Nile tilapia were grown out at the Demonstration Unit of Production in Net-Tanks of the State University of Maringá, Diamante do Norte, Paraná, Brazil.

In this study, Nile tilapia fingerlings were considered. The larvae went through the process of sexual reversion, and then 800 animals were randomly collected, distributed, and cultivated in 16 floating cages of $1 \mathrm{~m}^{3}$ at the Demonstration Unit of Floating Cages Production, from January to June 2017. The animals received basal (control) and basal ration plus $1.2 \%$ of All-G-Rich ${ }^{\circledast}$, in the last 45 days prior to slaughter. The diet was given twice daily throughout the 150-day of grown.

A total of 151 Nile tilapia were slaughtered with 150 days of cultivation. The Indian clove oil (eugenol) was employed as general anesthesia (dosage $184.26 \mathrm{mg} \mathrm{L}^{-1}$ ), followed by decapitation and perforation of the cranium ensuring the animal death. ${ }^{9}$ Then, the fillets were manually prepared, skinned, identified, and vacuum packed.

\section{NIR spectra}

NIR spectra of the fillets were acquired on the same day the fish were packed (at $25{ }^{\circ} \mathrm{C}$, before freeze and over the package, the package composition is nylon). The spectra were obtained over the package due to two reasons: (i) for collecting the spectra in the intact fillet (without grind) it is necessary a plastic film protecting the portable NIR instrument. To do the spectra without this plastic film it would be necessary to standardize the distance between the sample and the instrument, which would be more complicated in terms of reproducibility. (ii) It was performed a first-order multivariate calibration model (PLS). In this model type, it is possible the calibration in the interference presence (package). So, the plastic type is a part of method, once the plastic composition changes, the interferent may not be modeled.
A portable instrument MicroNIR ${ }^{\mathrm{TM}} 1700 \mathrm{JDSU}$ was used for the analysis of each fish fillet, the spectra acquisition was performed on the flesh side in three different points (Figure 1). All spectra were collected in diffuse reflectance (from 900 to $1600 \mathrm{~nm}$, step $4 \mathrm{~nm}, 32$ scans), and the spectra recorded for each sample have been averaged. After the NIR spectra collection, the points of collection were marked over the package using a pen, and the fillets were stored at $-18{ }^{\circ} \mathrm{C}$ until the beginning of the $\mathrm{n}-3$ and $\mathrm{n}-6$ analysis.

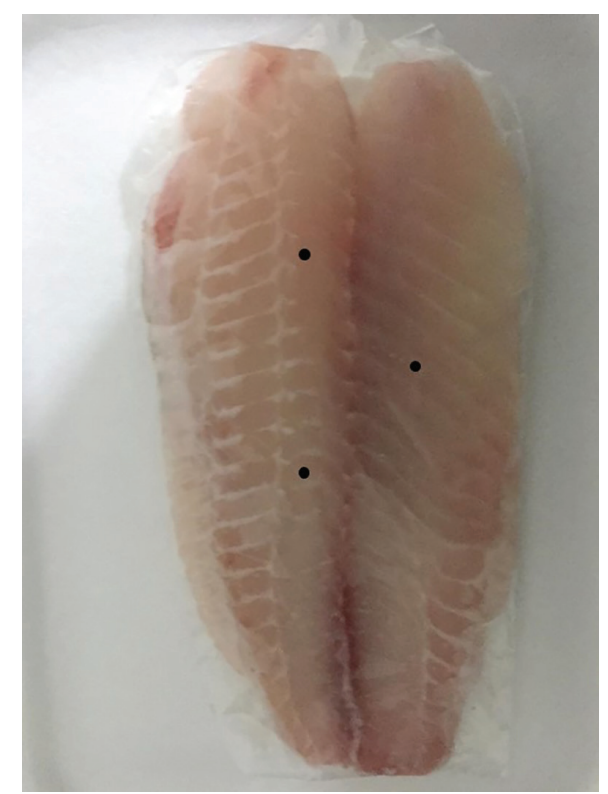

Figure 1. Photo of a fillet of Nile tilapia, vacuum packed, showing the points where NIR spectra were collected (black points).

This research was approved in accordance with the animal ethics committee of State University of Maringá, under the certificate number 8249200318 .

Lipid extraction

Total lipids were extracted according to Bligh and Dyer ${ }^{16}$ method. About $15 \mathrm{~g}$ of fillets were weighed. In a blender, thirty milliliters of methanol (Qhemis, São Paulo, Brazil) were added to the samples and stirred to homogenize the sample and open the micelles. After that $15 \mathrm{~mL}$ of chloroform (J.T. Baker, Phillipsburg, USA) was added and the solution was vigorously stirred for $5 \mathrm{~min}$. After this time, another $15 \mathrm{~mL}$ of chloroform was added, and the solution stirred for $2 \mathrm{~min}$. Finally, $15 \mathrm{~mL}$ of distilled water was added to the mixture and stirred for $5 \mathrm{~min}$. The obtained solution was vacuum filtered through a Büchner funnel with filter paper, and the remaining solution was transferred to a $250 \mathrm{~mL}$ separatory funnel. After the separation, the lower part, containing chloroform and lipids, were transferred to a $250 \mathrm{~mL}$ round bottom flask 
and the solvent was evaporated under reduced pressure at low temperature $\left(30^{\circ} \mathrm{C}\right)$.

\section{Sample derivatization}

Fatty acid derivatization was performed according to ISO $5509,{ }^{17}$ with modifications. Briefly, a solution of the internal standard methyl ester of tricosanoic acid (C23:0, purity $\geq 99.0 \%$, Sigma, St. Louis, USA), was prepared at a concentration of $1 \mathrm{mg} \mathrm{mL}^{-1}$ using iso-octane (Dinâmica, Indaiatuba, Brazil) as solvent. An aliquot of the internal standard solution $(250 \mu \mathrm{L})$ was transferred to an Eppendorf and the solvent was evaporated using $\mathrm{N}_{2}$. After dryness, approximately $20 \mathrm{mg}$ of sample was added to the Eppendorf and the exact mass was recorded to be used in the quantification.

For the esterification of the acylglycerols, $2.0 \mathrm{~mL}$ of iso-octane was used to solubilize the sample and the mixture was vortexed for $30 \mathrm{~s}$, after that $2.0 \mathrm{~mL}$ of potassium hydroxide (KOH, Dinâmica, Indaiatuba, Brazil) in methanol $\left(2 \mathrm{~mol} \mathrm{~L}^{-1}\right)$ was added and vigorously vortexed during $2.0 \mathrm{~min}$ at room temperature. After phase separation, the supernatant was collected for later gas chromatography analysis. The esterification was performed $24 \mathrm{~h}$ before the injection.

\section{Chromatographic analysis (GC-FID)}

\section{Instrumentation}

The analysis of fatty acid methyl esters (FAMEs) were performed on a gas chromatography, Shimadzu-2010 Plus AF (Shimadzu Co., Kyoto, Japan), coupled to a flame ionization detector (FID).

\section{Chromatographic conditions}

The FAMEs were separated in a fused silica capillary column $(60 \mathrm{~m} \times 0.22 \mathrm{~mm}$ i.d., $\times 0.25 \mu \mathrm{m}, 70 \%$ cyanopropyl polysilphenylene-siloxane, TR-FAME, Thermo Fisher Scientific, Waltham, USA). The flow of $\mathrm{H}_{2}$ (carrier gas) was $1.10 \mathrm{~mL} \mathrm{~min}^{-1}$ with $30.0 \mathrm{~mL} \mathrm{~min}^{-1}$

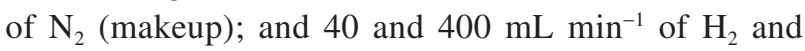
synthetic air, respectively, to the detector flame. The injection volume was $1 \mu \mathrm{L}$ using 1:40 split ratio. Injector and detector temperatures were set to 225 and $240{ }^{\circ} \mathrm{C}$, respectively. The oven temperature program started at $160{ }^{\circ} \mathrm{C}$ and was held at this temperature for $5 \mathrm{~min}$, then raised to $225^{\circ} \mathrm{C}$ at $4{ }^{\circ} \mathrm{C} \mathrm{min}^{-1}$ and kept at this temperature for $5 \mathrm{~min}$. Thus, the total run time was $26.25 \mathrm{~min}$. The identification of the FAMEs was made by comparison of the retention time of the compounds with the retention time of the authentic standards (FAME Mix, C4-C24,
Sigma, St. Louis, USA). Retention times and peak areas were determined using LabSolutions (GC Solution) software (Shimadzu Co., Kyoto, Japan).

\section{Quantification}

The absolute quantification of the methyl esters of fatty acids was carried out by internal standardization using tricosanoic acid methyl ester (C23:0, purity $\geq 99.0 \%$, Sigma, St. Louis, USA) as internal standard, according to Joseph and Ackman. ${ }^{18}$ To calculate the FAs concentration of tilapia fillet samples in $\mathrm{mg} \mathrm{g}^{-1}$ of total lipids, the theoretical correction factors for FID were used, ${ }^{19}$ according to equation 1:

$\mathrm{FA}=\frac{\mathrm{A}_{\mathrm{X}} \times \mathrm{W}_{\mathrm{IS}} \times \mathrm{CF}_{\mathrm{X}}}{\mathrm{A}_{\mathrm{IS}} \times \mathrm{W}_{\mathrm{X}} \times \mathrm{CF}_{\mathrm{AE}}} \times 100$

where: FA is the concentration of fatty acids in mg per $\mathrm{g}$ of total lipids; $A_{X}$ is the peak area (fatty acids); $A_{I S}$ is the peak area of internal pattern methyl ester of tricosanoic acid (C23:0); $\mathrm{W}_{\mathrm{IS}}$ is the mass of the internal pattern (in $\mathrm{mg}$ ) added to the sample; $\mathrm{W}_{\mathrm{X}}$ is the sample mass (in $\mathrm{mg}$ ); $\mathrm{CF}_{\mathrm{X}}$ is the theoretical correction factor determined based on the percentage by mass of active carbons in the molecule compared to the internal standard. The theoretical factors used were proposed by Visentainer. ${ }^{19} \mathrm{CF}_{\mathrm{AE}}$ is the conversion factor needed to express the results in $\mathrm{mg}$ of fatty acids instead of methyl esters.

Multivariate calibration

PLS regression was used as a tool for multivariate calibration. The PLS calculations were carried out with the PLS-Toolbox version 5.2. ${ }^{20}$ The outliers detection and figures of merit calculations were carried out with a homemade MATLAB ${ }^{21}$ routine.

PLS regression tool has been discussed in detail in relevant references. ${ }^{22,23}$ In this case, the data matrix $\mathbf{X}$ was constituted by the NIR spectra of the fillets of Nile tilapia samples and the vector $\mathbf{y}$ contained the reference values for $n-3$ or $n-6$, obtained from the chromatographic analysis. The spectra were preprocessed by the 'savgol' algorithm, ${ }^{24}$ through first derivative ( 7 points and first-order polynomial). The model was developed with mean center pre-processing for both $\mathbf{X}$ and $\mathbf{y}$.

\section{Results and Discussion}

First of all, an outlier assessment was performed on the data set. This evaluation was assessed through the plot of Hotelling $\mathrm{t}^{\wedge} 2$ against $\mathrm{Q}$ residuals (Figure $\mathrm{S} 1$ in the 
Supplementary Information). Samples with high Hotelling $\mathrm{t}^{\wedge} 2$ and $\mathrm{Q}$ residuals were removed from the data set resulting in a total of 138 samples.

The calibration and validation data sets had 103 and 35 samples, respectively, for n-3 and n-6 determination. The samples were selected by the 'kenston' algorithm. ${ }^{25}$ Figure 2 shows the spectra of all samples in the spectral range used in the models development. The spectra obtained from Nile tilapia without the package (mean of triplicate), and from the package (mean of triplicate) are also included in order to confirm that the modeled spectra profile refers to Nile tilapia itself.

Two models were built, one for $n-3$ and another for $\mathrm{n}-6$. The calibration and validation sets were, in both cases, evaluated for outliers identification based on data with extreme leverage, unmodeled residuals in spectral data and unmodeled residuals in property of interest, ${ }^{26}$ which in this case were $n-3$ or $n-6$.
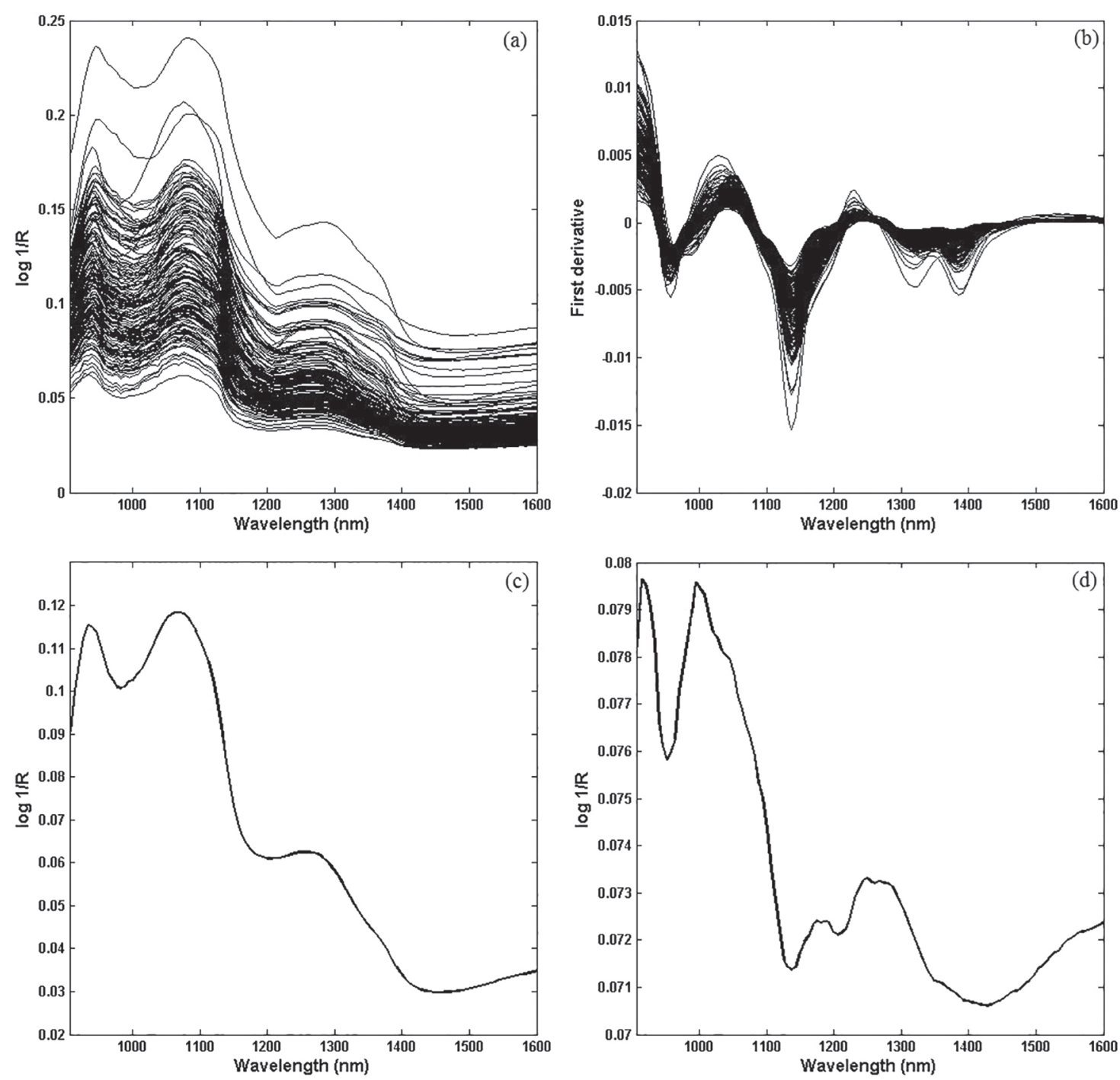

Figure 2. NIR spectra of fillets of Nile tilapia samples. (a) Raw spectra; (b) spectra after first derivative; (c) Nile tilapia without package; (d) package. 
sample. If a sample presents a difference between its reference value and its estimate larger than twice the RMSEC, this sample is an outlier. ${ }^{26}$

The outliers, identified by the previous procedures, were excluded, and this procedure resulted in 96 and 24 samples for calibration and validation, respectively, in the n-3 model determination. For the n- 6 model the outliers evaluation resulted in a total of 96 samples in the calibration set and 25 samples in the validation set.

The optimum model dimension was chosen by the root mean square error of cross-validation (RMSECV) for the calibration samples, obtained by contiguous block crossvalidation of ten samples. Furthermore, the percentage of explained variance in $\mathbf{y}$ was also considered. This procedure resulted in the choice of 14 latent variables (LVs) for meancentered model development for $\mathrm{n}-3$ and $17 \mathrm{LVs}$ for the model in predicting $\mathrm{n}-6$.

The parameters for model performance estimation (validation parameters), determined according to the equations presented in Santos et al., ${ }^{27}$ are shown in Table 1.

RMSEC and root mean square error of prediction (RMSEP) are accuracy indicatives (reporting the closeness of agreement between the reference value and the value found by the calibration model), ${ }^{26,28,29}$ and showed that models dimension, despite the high number of latent variables, was properly chosen, i.e., the models were not overfitted. Another way to confirm the achieved accuracy is the adjust or fit to the models, represented by the plot of the $n-3$ and n- 6 determined by reference method against n-3 and n- 6 determined by NIR/PLS model (Figure 3). This is important because sometimes RMSEC and RMSEP

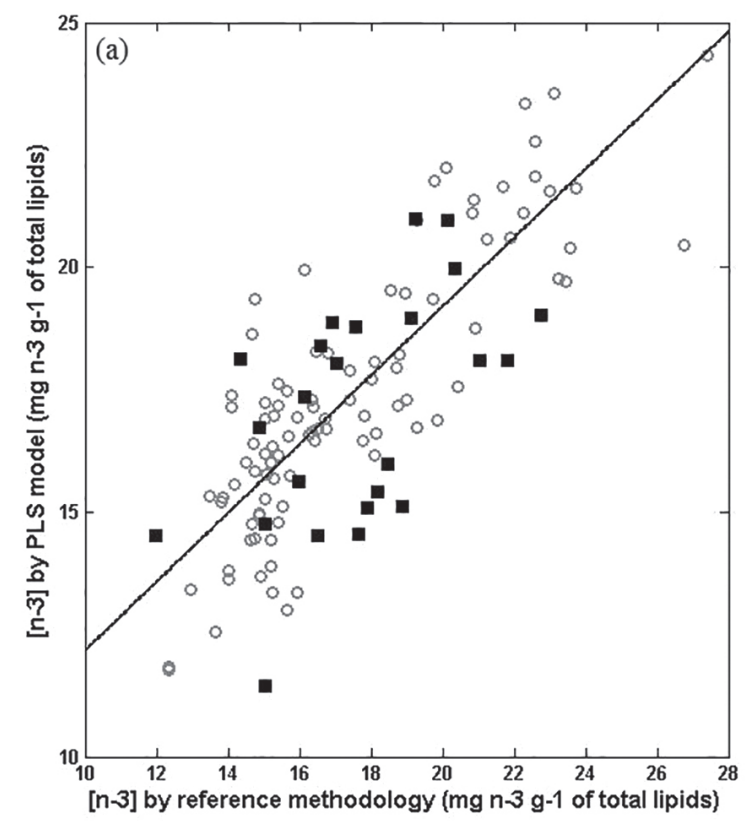

Table 1. Validation parameters of NIR/PLS models

\begin{tabular}{|c|c|c|c|}
\hline \multicolumn{2}{|c|}{ Parameter for model performance estimation } & \multirow[b]{2}{*}{$12.34-27.40$} & \multirow[b]{2}{*}{$93.93-145.81$} \\
\hline $\begin{array}{l}\text { Range calibrated / (mg FA g }{ }^{-1} \\
\text { of total lipids) }\end{array}$ & & & \\
\hline \multirow{2}{*}{$\begin{array}{l}\text { Accuracy / (mg FA g }{ }^{-1} \\
\text { of total lipids) }\end{array}$} & RMSEC & 1.90 & 4.73 \\
\hline & RMSEP & 2.39 & 4.76 \\
\hline $\begin{array}{l}\text { Analytical sensitivity }{ }^{-1} / \\
\text { (mg FA g } \text { g }^{-1} \text { of total lipids) }\end{array}$ & & 1.56 & 3.81 \\
\hline Correlation coefficient & & 0.7031 & 0.7077 \\
\hline $\begin{array}{l}\text { Limit of detection / } \\
\text { (mg FA g }{ }^{-1} \text { of total lipids) }\end{array}$ & & 5.14 & 31.31 \\
\hline $\begin{array}{l}\text { Limit of quantification / } \\
\text { (mg FA } \mathrm{g}^{-1} \text { of total lipids) }\end{array}$ & & 15.58 & 93.93 \\
\hline
\end{tabular}

FA: fatty acid; RMSEC: root mean square error of calibration; RMSEP: root mean square error of prediction.

parameters incorporate random and bias errors. The correlation coefficient value of 0.7031 for $\mathrm{n}-3$ and 0.7077 for $\mathrm{n}-6$ were considered satisfactory and in agreement with previous researches ${ }^{26,30,31}$ that reported coefficient value around 0.7 when the reference method is considered complex, with several sample preparation steps.

Accuracy can also be confirmed by the elliptical joint confidence regions. ${ }^{29}$ Figure 4 shows the elliptical joint confidence regions for $\mathrm{n}-3$ and $\mathrm{n}-6$ models. It is observed that the ellipse contains the ideal point (1.0) for slope and intercept, respectively, showing that the reference value and the NIR/PLS model are not significantly differencing at the $95 \%$ confidence level. This result allows concluding also, on the basis of the $95 \%$ confidence

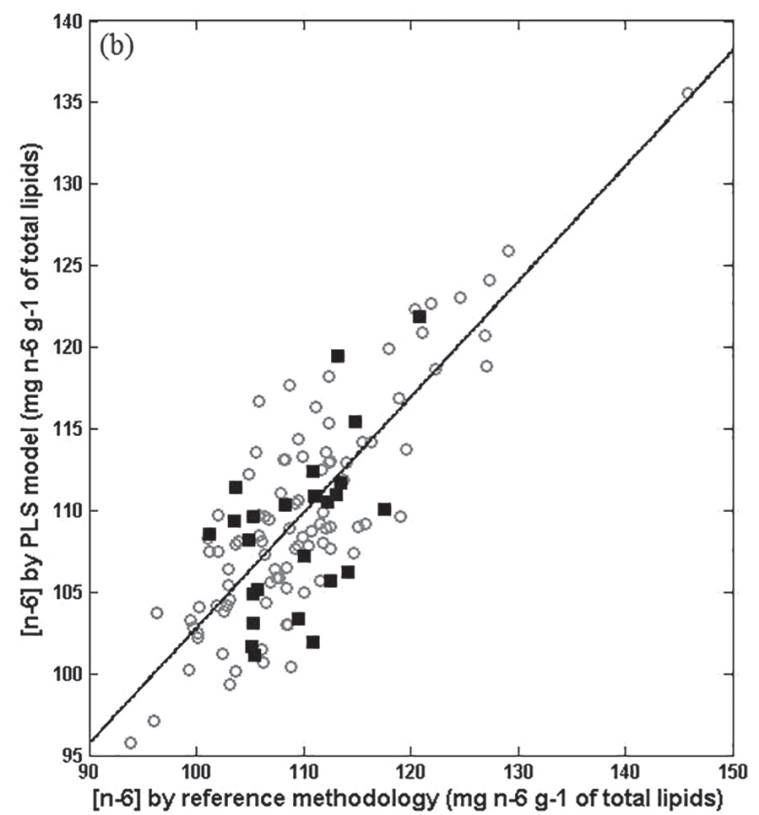

Figure 3. Reference values against the values estimated by the NIR/PLS model (a) for n-3; (b) for n-6. ( $\bigcirc$ ) Calibration samples and ( $\boldsymbol{\square}$ ) validation samples. 
intervals, that no constant or proportional systematic errors are present in the model since the intervals contain the expected values of 1 and 0 for the slope and the intercept, respectively. ${ }^{32,33}$

Due to the steps in PLS model development, the analytical sensitivity is more suitable for evaluating the sensitivity of a multivariate calibration model. Considering that spectrum noise represents the largest source of error, the inverse of the analytical sensitivity (or analytical sensitivity $^{-1}$ ) allows the establishment of a minimum

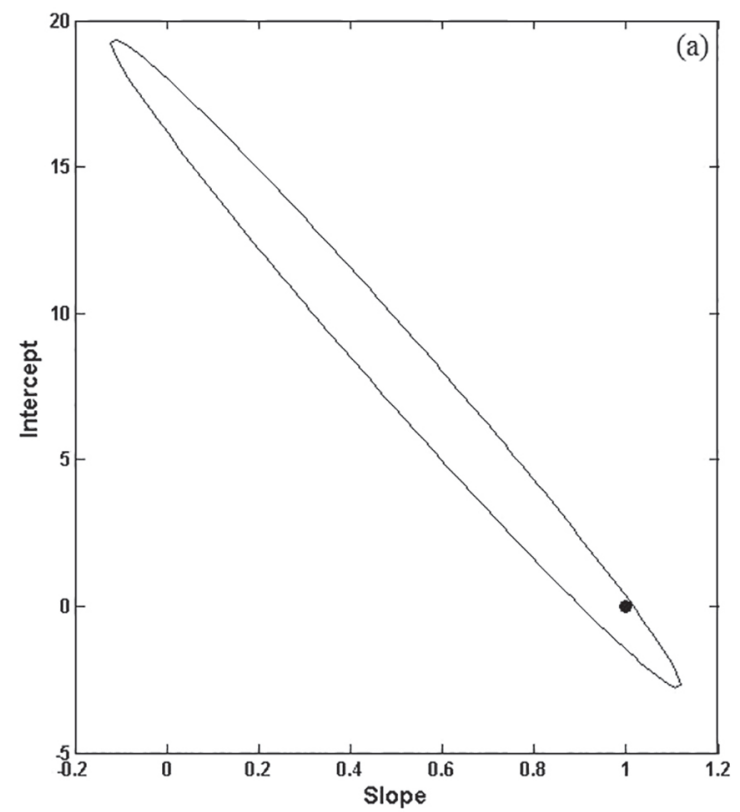

concentration difference, which is discernible by the model in the range of concentrations applied. Based on this, it is possible to distinguish samples with concentration difference of $1.56 \mathrm{mg} \mathrm{FA} \mathrm{g}^{-1}$ (n-3) of total lipids, and $3.81 \mathrm{mg} \mathrm{FA} \mathrm{g}^{-1}$ (n-6) of total lipids.

Limits of detection and quantification for the NIR/PLS models are in agreement with the measured quantities and the RMSEP obtained. Therefore, the NIR/PLS models are appropriate to quantify n-3 and n-6 in fillets of Nile tilapia, since their ranges are between 12.34 and $27.40 \mathrm{mg} \mathrm{FA} \mathrm{g}^{-1}$

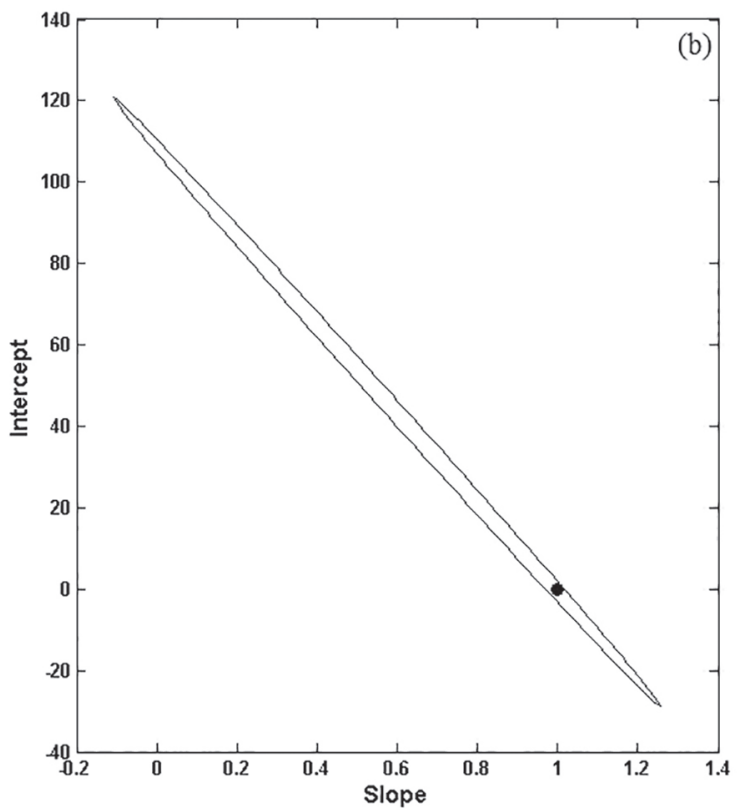

Figure 4. Elliptical joint confidence regions at $95 \%$ for the slope and intercept of the regression of predicted n-3 (a) and n-6 (b) against reference values by using ordinary least squares.
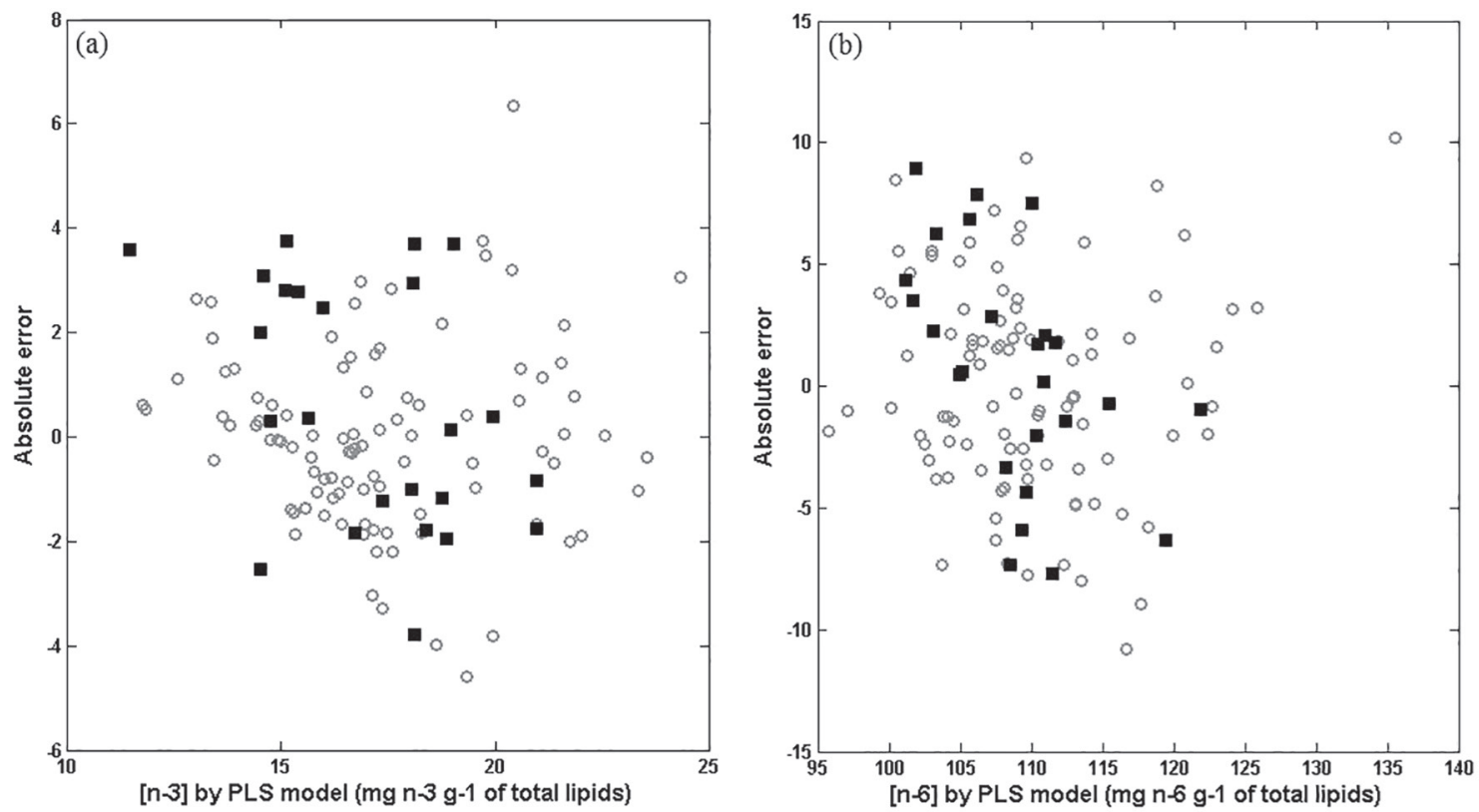

Figure 5. Residuals for (a) n-3; (b) n-6. (O) Calibration samples and ( validation samples. 
of total lipids, and between 93.93 and $145.81 \mathrm{mg} \mathrm{FA} \mathrm{g}^{-1}$ total lipid, respectively.

Residuals plot from calibration and validation samples are shown in Figure 5 and are linearity indicative for NIR/ PLS models. The random distribution of absolute errors is an expected behavior that suggests this data set fits into a linear model.

The results obtained for the parameters for model performance showed that the proposed method based on NIR/PLS can be promising. Furthermore, the spectra are acquired on the vacuum-packed fillet that contributes to the NIR/PLS method in terms of cost and quickness.

\section{Conclusions}

NIR spectroscopy coupled with the PLS model is suitable to determine omega- 3 and omega- 6 in the vacuumpacked fillet of Nile tilapia. The outliers were detected, and the models were validated by the parameters for model performance determination, showing promising results. The appropriate values achieved for accuracy, limits of detection and quantification indicates that the NIR/PLS models can be an alternative to chromatography to determine omega- 3 and omega-6 in fillets of Nile tilapia. Moreover, the NIR/PLS models present advantages compared to chromatographic methods. They do not require sample preparation and do not generate residues during the analysis, besides the capacity for being updated to other quality parameters of fillets of Nile tilapia.

\section{Supplementary Information}

Supplementary information is available free of charge at http://jbcs.sbq.org.br as PDF file.

\section{Acknowledgments}

The authors aknowledge CAPES (Coordenação de Aperfeiçoamento de Pessoal de Nível Superior) and Fundação Araucária (033/2019).

\section{Author Contributions}

Carlos A. L. de Oliveira, Makoto Matsushita and Ricardo P. Ribeiro were responsible for the conceptualization, formal analysis, funding acquisition and project administration; Jonathan C. Carvalho and Jailton S. Bezerra Junior for the data curation; Aline Coqueiro performed the quantification of fatty acids by gas chromatography; Dayane A. dos Santos, Aline Coqueiro, Thays R. Gonçalves, Paulo H. Março and Patrícia Valderrama for the data curation, software, validation, visualization and writing original draft; Patrícia Valderrama, Paulo H. Março, Aline Coqueiro and Ricardo P. Ribeiro for the writing review and editing.

\section{References}

1. Suemitsu, L.; Cristianini, M.; LWT - Food Sci. Technol. 2019, 101, 92 .

2. Monteiro, M. L. G.; Mársico, E. T.; Lázaro, C. A.; Canto, A. C. V. C. S.; Lima, B. R. C. C.; da Cruz, A. G.; Conte-Júnior, C. A.; J. Food Sci. Technol. 2015, 52, 2598.

3. Food and Agriculture Organization of the United Nations (FAO); The State of World Fisheries and Aquaculture 2018 - Meeting the Sustainable Development Goals; FAO: Rome, 2018. Available at http://www.fao.org/3/i9540en/i9540en.pdf, accessed in April 2020.

4. Raatz, S. K.; Silverstein, J. T.; Jahns, L.; Picklo, M. J.; Nutrients 2013, 5, 1081.

5. Béné, C.; Arthur, R.; Norbury, H.; Allison, E. H.; Beveridge, M.; Bush, S.; Campling, L.; Leschen, W.; Little, D.; Squires, D.; Thilsted, S.H.; Troell, M.; Williams, M.; World Dev. 2016, 79, 177.

6. Can, M. F.; Günlü, A.; Can, H. Y.; Food Sci. Technol. 2015, 35 , 339.

7. Martin, C. A.; Almeida, V. V.; Ruiz, M. R.; Visentainer, J. E. L.; Matsushita, M.; Souza, N. E.; Visentainer, J. V.; Rev. Nutr. 2006, 19, 761.

8. Shahidi, F. In Omega-3 Oils: Applications in Functional Foods; Hernandez, E. M.; Hosokawa, M., eds.; Elsevier: London, UK, 2011, ch. 1

9. Vidal, L. V. O.; Albinati, R. C. B.; Albinati, A. C. L.; Lira, A. D.; Almeida, T. R.; Santos, G. B.; Pesqui. Agropec. Bras. 2008, 43, 1069 .

10. Baqueta, M. R.; Coqueiro, A.; Valderrama, P.; J. Food Sci. 2018, 84, 1247.

11. Baqueta, M. R.; Coqueiro, A.; Março, P. H.; Valderrama, P.; Food Anal. Methods 2020, 13, 50.

12. Pereira, J. M.; Leme, L. M.; Perdoncini, M. R. F. G.; Valderrama, P.; Março, P. H.; Food Anal. Methods 2018, 11, 1878.

13. Leme, L. M.; Montenegro, H. R.; Santos, L. R.; Sereia, M. J.; Valderrama, P.; Março, P. H.; Food Anal. Methods 2018, 11, 1944.

14. Rosa, L. N.; Coqueiro, A.; Março, P. H.; Valderrama, P.; Food Chem. 2019, 273, 52.

15. Santos, L. R.; Zangirolami, M. S.; Silva, N. O.; Valderrama, P.; Março, P. H.; Pesq. Agropec. Bras. 2018, 53, 97.

16. Bligh, E. G.; Dyer, W. J.; Can. J. Biochem. Physiol. 1959, 37, 911.

17. International Organization for Standardization (ISO); ISO 5509: Animal and Vegetable Fats and Oils - Preparation of Methyl Esters of Fatty Acids; ISO: London, 1978, p. 01-06.

18. Joseph, J. D.; Ackman, R. G.; J. AOAC Int. 1992, 75, 488.

19. Visentainer, J. V.; Quim. Nova 2012, 35, 274. 
20. PLS-Toolbox, version 5.2; Eigenvectors Research, Manson, WA, USA, 2009.

21. MATLAB, version R2007b; Mathworks, Natick, MA, USA, 2007.

22. Geladi, P.; Kowalski, B. R.; Anal. Chim. Acta 1986, 185, 1.

23. Brereton, R. G.; Analyst 2000, 125, 2125.

24. Savitzky, A.; Golay, M. J. E.; Anal. Chem. 1964, 36, 1627.

25. Kennard, R. W.; Stone, L. A.; Technometrics 1969, 11, 137.

26. Valderrama, P.; Braga, J. W. B.; Poppi, R. J.; J. Agric. Food Chem. 2007, 55, 8331.

27. Santos, D. A.; Lima, K. P.; Março, P. H.; Valderrama, P.; J. Braz. Chem. Soc. 2016, 27, 1912.

28. Martens, H.; Naes, T.; Multivariate Calibration; Wiley: New York, 1989.
29. Valderrama, P.; Braga, J. W. B.; Poppi, R. J.; Quim. Nova 2009, 32, 1278.

30. Ferreira, D. S.; Pallone, J. A. L.; Poppi, R. J.; Food Res. Int. 2013, 51, 53.

31. Santos, D. A.; Lima, K. P.; Cavalcante, V.; Coqueiro, A.; Consolin, M. F. B.; Consolin Filho, N.; Março, P. H.; Valderrama, P.; Food Anal. Methods 2018, 11, 1915.

32. Riu, J.; Rius, F. X.; Anal. Chem. 1996, 68, 1851.

33. Valderrama, P.; Romero, A. L.; Imamura, P. M.; Magalhães, I. R. S.; Bonato, P. S.; Poppi, R. J.; Anal. Bioanal. Chem. 2010, 397, 181.

Submitted: October 3, 2019

Published online: May 5, 2020 